\title{
Historia
}

\section{Joseph Barcroft y la expedición anglo-americana a los Andes Peruanos (1921-1922)}

\author{
Oscar G. Pamo ${ }^{1,2}$
}

Resumen

Joseph Barcroft (1872-1947), fisiólogo británico de Cambridge, vino al Perú hacia fines de 1921 liderando la expedición angloamericana para estudiar las características fisiológicas que permiten a los humanos aclimatarse a la vida en las grandes alturas. Arribaron a Cerro de Pasco, realizando diversas mediciones y a diferentes altitudes, en ellos, en el personal norteamericano de la mina y en algunos nativos. Esta experiencia, publicada dos años más tarde, generaría en el doctor Carlos Monge Medrano (1884-1970) y otros investigadores nacionales el interés de conocer la biología y la patología del hombre andino, en 1927.

Palabras clave Historia de la medicina, Perú; altitud; ecosistema andino; Barcroft, Joseph.

\section{Joseph Barcroft and the Anglo-American expedition to the Peruvian Andes (1921-1922)}

\section{Abstract}

Joseph Barcroft (1872-1947), British Cambridge physiologist, came to Peru at the end of 1921 leading the Anglo-American expedition in order to study the physiological characteristics that let human beings acclimatize to live at high altitude environments. They arrived to Cerro de Pasco, carrying out several measurements at different altitudes, among themselves, the Mining Co.'s American personnel and some native dwellers. This survey published as a paper two years later would arouse in 1927 Dr. Carlos Monge-Medrano (18841970) and other local investigators the interest in searching for the biology and pathology of the Andean people.

Keywords: History of medicine, Peru; altitude; Andean ecosystem; Barcroft, Joseph.

El nombre de Joseph Barcroft está ligado indirectamente a la historia de la medicina peruana. Él

1 Médico Internista. Departamento de Medicina, Hospital Arzobispo Loayza. Lima, Perú.

2 Profesor Principal. Departamento de Medicina, Facultad de Medicina Alberto Hurtado, Universidad Peruana Cayetano Heredia. Lima, Perú. lideró la expedición angloamericana que a fines de 1921 vino a los Andes peruanos a estudiar la fisiología respiratoria del humano sometido a baja presión atmosférica. Sus conclusiones, que publicó dos años después, dieron lugar a una respuesta del Dr. Carlos Monge Medrano en 1927. De allí en adelante, se realizó numerosas investigaciones para estudiar la salud y la enfermedad del habitante de las grandes alturas en nuestro país.

\section{DE LOS ANTECEDENTES}

La gran discusión científica y filosófica que empezó a fines del siglo XIX fue si el oxígeno entraba a la sangre por simple difusión o era secretado activamente por las células alveolares. Fick, desde un punto de vista netamente mecanicista y con sustento matemático, sostuvo que el pasaje ocurría por difusión. ${ }^{1}$ Ludwig, desviándose de su materialismo mecanicista, sostuvo que

\footnotetext{
${ }^{1}$ Adolph Fick (1829-1901). Fisiólogo alemán. Discípulo de Ludwig, fue profesor de Fisiología en Würtzburg. Debido a sus conocimientos de física y matemáticas, introdujo los métodos cuantitativos para los procesos biológicos. Escribió 'Física Médica', formuló la difusión de los gases a través de las membranas biológicas y midió el gasto cardíaco.
} 
la difusión del oxígeno, y también del $\mathrm{CO}_{2}$, podría ser facilitado por los pulmones. ${ }^{2}$

Veinte años más tarde, Bohr, uno de los discípulos sobresalientes de Ludwig, realizando mediciones con su hemoaerotonómetro, obtuvo evidencias que apoyaban el concepto de un transporte activo de oxígeno por las células alveolares. ${ }^{3}$

En 1903, Krogh, discípulo de Bohr, hizo su tesis doctoral utilizando como hipótesis de trabajo el rol secretor de oxígeno por los pulmones. ${ }^{4}$ Con su microaerotonómetro, Krogh obtuvo resultados que obedecían a los principios matemáticos de Fick sobre la difusión de los gases. En los siguientes quince años, publicó una serie de trabajos dando a conocer sus aportes y modelos matemáticos sobre la difusión del oxígeno y la oxigenación de los tejidos; y estableció que el oxígeno también pasaba de los capilares a los tejidos por simple difusión.

Por otro lado, en 1895, Haldane y su colega JL Smith habían realizado diversas innovaciones para medir los gases generados y consumidos en el organismo; en especial, desarrolló un método indirecto utilizando el CO para medir la concentración de oxígeno en la sangre. ${ }^{5}$ Como el CO compite con el oxígeno para fijarse a la hemoglobina, a mayor concentración de oxígeno en la sangre menor será la capacidad fijadora de CO.

En 1905, Haldane demostró el mecanismo de retroalimentación regulado por el sistema nervioso y por el cual un aumento de la concentración de $\mathrm{CO}_{2}$ alveolar producía un incremento de la frecuencia respiratoria. Por ende, era posible también asumir que las células alveolares ejercían un control sobre la concentración de oxígeno en la sangre; es decir, un efecto secretor (o de transporte activo) toda vez que disminuyera la concentración de oxígeno en el aire alveolar.

En 1911, Haldane, al procesar los resultados de sus mediciones en el Monte Pikes, Colorado, encontró que, a pesar de la disminución de la concentración de oxígeno en el aire inspirado, la sangre se mantenía oxigenada adecuadamente. Por ello concluyó que, en las alturas la concentración de oxígeno en la sangre excedía la existente en el aire alveolar. Esto apoyaba la teoría del rol secretor de oxígeno de los pulmones.

Hacia 1920, los trabajos de Krogh, la simple difusión del oxígeno, estaban contra la posición de Ludwig-BohrHaldane, papel secretor de oxígeno de los pulmones, pugna que se denominó la "gran controversia de la secreción de oxígeno" $\left.{ }^{(}\right)$. Fue allí que intervino la figura de Barcroft.

\section{BARCROFT FISIÓLOGO}

Segundo de cinco hermanos, Joseph Barcroft nació el 26 de julio de 1872, en The Glen, Newry, County Down, Irlanda. Sus padres, Henry Barcroft y Anna Richardson Malcolmson, fueron cuáqueros originarios de Lancashire y descendientes de antiguas y acomodadas familias $\left(^{2}\right)$ (Figura 1).

Realizó sus estudios en la Friend's School de Bootham, York, entre 1884 y 1888, destacando como un excelente alumno, donde se reveló su interés por las ciencias naturales.

En el verano de 1888, ingresó a estudiar en la Escuela Leys de Cambridge y fue aleccionado para estudiar simultáneamente en la Universidad de Londres, en 1889. En 1891, se graduó como Bachiller en Ciencias de esta universidad, cuando aún no finalizaba los estudios de la Escuela de Leys.

En octubre de 1893, ingresó al King's College de Cambridge para estudiar Fisiología,. Tras una brillante carrera, recibiendo honores, desde estudiante, en ciencias naturales, se graduó como Bachiller en Artes en 1896. Regresó al Laboratorio de Fisiología e inició la que sería su primera investigación, el metabolismo gaseoso de la glándula submaxilar del perro, tema sugerido por el

\footnotetext{
2 Karl Friedrich Wilhelm Ludwig (1816-1895). Primer profesor de Fisiología de la Universidad de Leipzig. Profesor de anatomía y fisiología de Marburg, Zurich y Viena. Fundó el Instituto de Fisiología de Leipzig.

3 Christian Bohr (1855-1911). Fisiólogo danés. Profesor de Fisiología en la Universidad de Copenhagen, en 1886. Padre del físico Niels Bohr (1885) y del matemático Harald Bohr (1887).

4 Schack August Steenberg Krogh (1874-1949). Fisiólogo danés. Por sus trabajos sobre la circulación capilar y la oxigenación de los tejidos le fue conferido el Premio Nobel en 1920. Introdujo los radioisótopos en la investigación biológica y estudió la fisiología del ejercicio.

5 John Scott Haldane (1860-1936). Fisiólogo británico. Estudió los sistemas respiratorios, creó la cámara que lleva su nombre para el estudio del intercambio gaseoso y el metabolismo de los animales.
} 


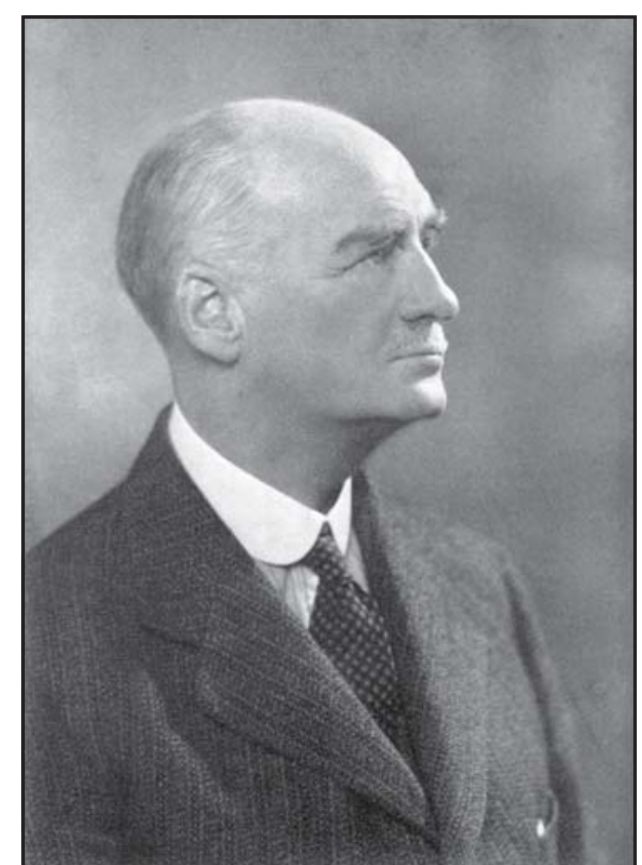

Figura 1. Dr. Joseph Barcroft (1872-1947).

profesor Langley y que lo terminaría en 1901. La primera dificultad que tuvo fue la de mejorar los aparatos de medición que se empleaba en ese entonces.

Cuando en 1899 fue admitido para el Fellowship del King's College, su disertación fue "Una investigación del metabolismo gaseoso de las glándulas salivales", cuyo texto había sido examinado por Langley y Haldane. En este mismo año, junto con HHW Pearson, ganó la Medalla Walshingham para investigación biológica y compartió con HH Dale el Premio Gedge para investigación fisiológica, en 1900.

En 1901, fue designado conferencista anual en ciencias naturales en el King's College, cargo que desempeñaría hasta 1909.

En 1902, junto con Robert S Ball Jr y JS Haldane, publicó un nuevo método para medir el oxígeno y el ácido carbónico en pequeñas cantidades. A pesar de no lograr la Cátedra Dunville de Fisiología en el Queen's College de Belfast, pues la fue concedida al fisiólogo italiano Angelo Mosso, Barcroft fue reconocido por sus trabajos.
En 1903, Sir Michael Foster dejó la Cátedra de Fisiología de Cambridge, que ocupaba desde 1883, y le sucedió John Newport Langley. ${ }^{6}$ En ese año, Barcroft se casó con Mary Agnetta Ball, hija del renombrado astrónomo Sir Robert Stawell Ball.

En 1904, nació su primogénito, Henry Barcroft, quien también sería fisiólogo, y después nació Robert Ball Barcroft, quien se dedicaría a la carrera militar. En ese año publicó sobre el metabolismo gaseoso del riñón y el intercambio de oxígeno del páncreas.

A fines de julio de 1905 partió hacia Cape Town, Sudáfrica, donde tendría lugar el Congreso de la Asociación Británica. Retornó vía el Canal de Suez y tuvo la oportunidad de conocer el Egipto.

Barcroft y col realizaron estudios sobre la utilización del oxígeno por los tejidos musculares de los mamíferos, mientras que WM Fletcher y FG Hopkins lo hicieron con los efectos de la condiciones aeróbicas y anaeróbicas en el músculo de la rana.

En 1907, presentó una comunicación preliminar sobre la naturaleza y velocidad de la sangre emergente de la glándula submaxilar del gato durante la estimulación del nervio simpático cervical y sobre el metabolismo gaseoso del corazón. En marzo de ese año, se le designó responsable de las clases prácticas y las demostraciones. Y publicó sobre las propiedades anticoagulantes del extracto purificado de sanguijuelas (hirudina).

En 1908, dio a conocer su método para el análisis de los gases sanguíneos e introdujo una técnica que estaría permanentemente asociada a su nombre o, en su forma más delicada, con el método micrométrico de Warburg. El objetivo fue reducir la probabilidad de error en las determinaciones comparativas de los contenidos de gases en las muestras de sangre arterial y venosa de los órganos, simplificando las engorrosas operaciones. Publicó el método del ferrocianuro para las determinaciones de los gases sanguíneos con propósitos clínicos. Su trabajo más importante en ese año fue una extensa revisión sobre el metabolismo gaseoso por los diferentes órganos.

En el Congreso de la Asociación Británica en Winnipeg, hacia fines de agosto de 1909, donde Barcroft estuvo ausente, se mencionó que él se hallaba comprometido en el estudio de ciertas condiciones

6 Michael Foster (1836-1907). Primer Profesor de Fisiología en Cambridge. Realizó notables aportes en biología, embriología, fisiología y medicina experimental. Escribió sobre la biografía de Claude Bernard y la fisiología de los siglos XVI y XVII. 
sanguíneas que influenciaban la respiración en las grandes alturas.

En marzo de 1910, se dirigió a Tenerife (en las Islas Canarias) para unirse a la expedición organizada por el profesor Gottfried von Pannwitz, de Berlín. ${ }^{7}$ La expedición estuvo conformada por los profesores Nathan Zuntz $^{8}$ (quien había invitado a Barcroft) y Carl Neuberg, de Berlín, Arnold Durig y Hermann von Schroetter, de Viena, C Gordon Douglas, de Oxford, y, Barcroft. Estuvieron del 21 de marzo hasta el 14 de abril, ascendiendo hasta la cabaña Alta Vista, unos 11000 pies (3 350 m), al sur del Monte Tenerife. Barcroft halló que la curva de disociación de la hemoglobina permanecía inalterada a nivel del mar, a 7000 pies y a 11000 pies, mientras que descendía la presión parcial de $\mathrm{CO}_{2}$. De acuerdo a la predicción de Bohr, la afinidad de la hemoglobina por el oxígeno debió incrementarse, pero ello no sucedió. Barcroft postuló que debería haber alguna otra sustancia que produjera el efecto observado y empezó los estudios con el ácido láctico, cuya producción se incrementaba en situaciones de asfixia y que también desviaba la curva a la derecha. ${ }^{9}$

Para aclarar sus dudas, especialmente si el ejercicio (escalamiento) producía resultados diferentes, Barcroft organizó la expedición hacia la zona montañosa de Italia. Con la ayuda de fondos de la Real Sociedad y de la Asociación Británica, se llevó a cabo la expedición hacia Monte Rosa. El grupo estuvo conformado por Barcroft, M Camis, GC Mathison, F Roberts y JH Ryffel. Con las facilidades brindadas por el profesor Vittorio Aducco de la Universidad de Pisa, esta vez alcanzaron hasta la cabaña Regina Margherita, cerca de 15000 pies. Entre sus conclusiones, complementadas más tarde con los resultados de su ascenso a Carlingford ${ }^{10}$ y los de GC Mathison, en Sugar Loaf (Gales), ambos a 1000 pies y en 1912, tenemos que en las alturas el $\mathrm{CO}_{2}$ disminuía en la sangre; que ésta se tornaba acidótica; que, probablemente, el ácido láctico producía la acidosis en reposo y en actividad física; y, que esta acidosis por sí sola no explicaría los cambios físicos y mentales, que tal vez ocurriría agregación de la hemoglobina y de otras moléculas. ${ }^{11}$

Es de resaltar el interés que por ese entonces se había generado entre los fisiólogos por los estudios de las propiedades de la sangre y la respiración en las grandes alturas. La razón radicaba en la «gran controversia de la secreción de oxígeno» por las células alveolares. En ese mismo año, se llevó a cabo la primera expedición angloamericana para estudios fisiológicos por JS Haldane y C Gordon Douglas, de la Universidad de Oxford, y Yandell Henderson y Edward C Schneider de la Universidad de Yale. Ellos estudiaron la adaptación fisiológica del humano sometido a baja presión atmosférica, en el laboratorio del Monte Pikes, Colorado, EEUU, a 14 000 pies $(4300 \mathrm{~m})$, donde midieron la presión alveolar del $\mathrm{CO}_{2}$, la frecuencia respiratoria, la concentración de hemoglobina y la aclimatación. Como se mencionó antes, Haldane encontró que la concentración de oxígeno se mantenía elevada relativamente a pesar de su disminución en el aire inspirado en la altura.

Hacia fines de 1911, Barcroft publicó sobre la formación y estimación de la metahemoglobina. A

\footnotetext{
7 Desde el primer tercio del siglo XIX, existía el interés por la acción benéfica de la luz solar sobre la tuberculosis y las enfermedades pulmonares. En 1910, en Bruselas, en la Novena Conferencia Internacional contra la Tuberculosis, se recomendó realizar expedición a las Cañadas (Tenerife) para estudiar dicho efecto. Esto se aprovechó para realizar otros estudios y mediciones.

8 Nathan Zuntz (1847-1920). Fisiólogo alemán. Realizó importantes contribuciones en metabolismo, fisiología de altura, ejercicio. Es considerado pionero de la medicina aeroespacial.

9 Actualmente, se conoce que en las alturas la curva de disociación de la hemoglobina se desvía a la derecha; es decir, aumenta la afinidad de la hemoglobina por el oxígeno. Los hallazgos de Barcroft serían explicables, en todo caso, por defectos técnicos y limitaciones de los aparatos de medición.

${ }^{10}$ En agosto de 1912, Barcroft realizó estudios de la curva de disociación de la hemoglobina a 1000 pies, en Carlingford Mountain, Irlanda, los que repetiría en el año siguiente.

${ }^{11}$ Cuando disminuye la presión parcial de oxígeno en el aire alveolar, por la hipoxia ambiental, se incrementa la presión parcial de $\mathrm{CO}_{2}$. Esto produce un aumento de la frecuencia respiratoria con la consiguiente disminución del $\mathrm{CO}_{2}$ (alcalosis respiratoria). Luego de dos o tres días, ocurre la compensación renal, eliminándose aniones bicarbonato (acidosis metabólica). Tanto la alcalosis respiratoria como la acidosis metabólica están compensadas; es decir, el pH sanguíneo es normal. Por otro lado, aumenta el gasto cardíaco, que se normaliza en pocos días, se incrementa la permeabilidad capilar y se incrementa el 2,3 difosfoglicerato, que diminuye la afinidad de la hemoglobina para facilitar su entrega a nivel tisular. La eritrocitosis inducida por la hipoxia es una respuesta tardía. ${ }^{(3-4)}$
} 
mediados de 1912, publicó sobre el incremento de la perfusión sanguínea al tejido glandular salival que no producía un incremento de la captación de oxígeno; y, que sí ocurría con la corta liberación de adrenalina. En octubre de ese año, publicó que el consumo de oxígeno se incrementaba de tres a cinco veces durante la alimentación y que el flujo sanguíneo de la arteria hepática era la fuente principal de oxígeno del hígado.

En enero de 1913, Barcroft y col presentaron el reporte preliminar, durante el Congreso de la Sociedad de Fisiología realizado en el King's College, en Londres, sobre el efecto del ejercicio, la altura, la dieta sin carbohidratos y el calor húmedo sobre la curva de disociación de la sangre. Junto con A Cookes realizaron la determinación de la saturación de la sangre arterial, usando el método de la punción arterial por primera vez. En los años siguientes, Barcroft investigó sobre diversos aspectos de la curva de disociación de la hemoglobina, su relación con el ácido carbónico y el efecto producido por la altitud.

Al iniciarse la Primera Guerra Mundial, continuó trabajando, aunque con muchas dificultades. Cuando los alemanes empezaron a usar gases, a mediados de 1915, fue enviado a Boulogne (Francia) a estudiar a los soldados afectados y luego pasaría a dirigir la Sección de Fisiología de la estación de Guerra Química del Gobierno en Porton, cerca de Salisbury. Barcroft realizó estudios experimentales con cloro y fosgeno en animales. Incluso, llevó a cabo un autoexperimento sobre la toxicidad del ácido prúsico, encerrándose en una cámara en la que se vertió dicho gas.

Terminada la guerra, Barcroft retornó al laboratorio universitario y a las actividades docentes. Hacia fines de 1919, dio a conocer sus experimentos en cabras sobre problemas circulatorios debido al envenenamiento gaseoso. Concluyó que, sucesivamente, se producía destrucción del endotelio capilar pulmonar, trombosis, isquemia y edema intersticial e intraalveolar. Que, sorpresivamente, la oxigenación de la sangre y la presión de la arteria pulmonar estuvieron dentro de límites normales y recomendaba no someterse a esfuerzo alguno en estas condiciones para evitar una descompensación. $\mathrm{Al}$ año siguiente publicó su trabajo sobre el tratamiento de los casos crónicos de envenenamiento gaseoso con la administración continua de oxígeno en cámaras.

Los estudios que realizó Barcroft en el Monte Tenerife y en Monte Rosa fueron sobre el efecto de la actividad física sobre la curva de disociación de la hemoglobina; pero, en 1920, decidió participar en la "gran controversia".

En febrero de 1920, llevó a cabo su famoso "experimento de la caja de vidrio", completamente sellada, en la que se introdujo y permaneció durante seis días. Hizo mediciones en reposo y en ejercicio. Para determinar el contenido de oxígeno en la sangre, no utilizó el método indirecto del $\mathrm{CO}$ de Haldane, sino el método directo aerotonométrico con la sangre obtenida por punción arterial ${ }^{12} ; \mathrm{y}$, midió la concentración del oxígeno alveolar en el aire exhalado en espiración máxima. De esta manera, pudo determinar el gradiente de oxígeno alveolar-arterial. Al consumir el oxígeno del ambiente interno, la frecuencia de su pulso se incrementó, presentó cefalea y una manifiesta incapacidad para concentrarse. El oxígeno ambiental disminuyó, a medida que lo consumía, hasta $84 \mathrm{~mm} \mathrm{Hg}$, correspondiente a una altitud de 18000 pies. Sus mediciones mostraron que la sangre arterial contenía menos oxígeno, en reposo y en actividad, que el aire alveolar, a temperatura corporal. Con esto demostró que el pulmón no secretaba oxígeno como había sido sostenido por Ludwig-Bohr-Haldane y le daba la razón a la tesis de Fick-Krogh. Cuando Barcroft presentó estos resultados en la Sociedad de Fisiología, Haldane contestó más tarde publicando que Barcroft «no se había aclimatado» y cuestionó sus resultados por no haber utilizado su método.

En agosto de 1920, en el Congreso de la Asociación Británica, en Cardiff, dio su conferencia sobre anoxemia, indicando que en casos extremos de anoxemia aguda ocurriría daño variable del sistema nervioso. Y, con respecto a la anoxemia crónica, sostuvo que por encima de los 18000 pies, donde vive y trabaja cierta gente, ocurría un proceso de compensación, que se le conocía como aclimatación, que era conseguido a expensas de la reserva funcional corporal; $y$, que la respiración en reposo semejaba a la del ejercicio a menor altura. Que, mientras que la anoxemia aguda simulaba la intoxicación

${ }^{12}$ En 1912, el científico alemán Hurter realizó por primera vez la punción arterial en cuatro pacientes. En 1918, Stadie realizó punciones arteriales en 90 pacientes con neumonía. Barcroft empleó la técnica de Stadie que suponía descubrir la arteria radial y canularla para obtener muestras de sangre. 
alcohólica, la anoxemia crónica simulaba la fatiga. Distinguió tres tipos de anoxemia: anóxica, anémica y congestiva. ${ }^{13}$

En enero de 1921, publicó su trabajo experimental sobre la anoxemia como un factor en el envenenamiento gaseoso con cloro y fosgeno. Durante los primeros meses de este año, consolidó los preparativos para su expedición a los Andes peruanos.

La expedición estuvo preparada desde inicios del verano y fue posible gracias al aporte económico de una docena de fuentes, especialmente de las instituciones a las cuales estaban ligados los investigadores: el Departamento de Química Física y la Fundación Proctor de la Universidad de Harvard; la Fundación Elizabeth Thompson; el Instituto Rockefeller de Investigaciones Médicas de Nueva York; la Universidad de Columbia (con los fondos aportados por Walter B James, Cleveland H Dodge y un anónimo); la Real Sociedad de Londres; una beca de investigación del Departamento de Fisiología de la Universidad de Toronto; las Fundaciones Moray y Carniege de Edimburgo; Sir Robert Hadfield y Sir Peter Mackie.

El objetivo de la expedición fue el de investigar las alteraciones, reales o supuestas, en el organismo que lo hacen adaptarse a las condiciones de vida en las grandes alturas. La edad de los expedicionarios varió entre 25 y 49 años, siendo Barcroft el mayor.

\section{LA EXPEDICIÓN A LOS ANDES PERUANOS}

El 17 de noviembre de 1921, Barcroft se embarcó en Liverpool en el buque SS Victoria de la Pacific Steam Navigation Company con destino al Perú, acompañado del profesor JC Meakins de Edimburgo y de JH Doggart del King's College. Para cumplir con su objetivo, se había elegido la zona de las minas de cobre, que se encontraban a no menos de 16000 pies, en la parte central de los Andes peruanos, porque era un buen lugar para el estudio. Posteriormente, Barcroft sostuvo que eligió el Perú por las facilidades (transporte, agua, electricidad) que había en todo nivel de las alturas y que iba a contar con por lo menos tres grupos de estudio bien diferenciados: los nativos que ancestralmente vivían en la zona, los operarios e ingenieros norteamericanos que trabajaban en las minas por varios años y los miembros de la expedición.

El grupo norteamericano había partido el 16 de noviembre en el SS Ebro y arribó al puerto del Callao tres semanas antes que los británicos. Estuvo integrado por CA Binger, del Instituto Rockefeller de Investigaciones Médicas, Nueva York; AV Bock, del Hospital General de Massachussetts; HS Forbes, de la Escuela Médica de Harvard; G Harrop, del Hospital Presbiteriano de Nueva Cork, y AC Redfield, de la Escuela Médica de Harvard.

El grupo británico pasó por La Habana el 7 de diciembre y cruzó el Canal de Panamá el 12 del mismo mes. Llegó al Callao a las 6:00 pm del 17 de diciembre, siendo recibido por varias embarcaciones pequeñas, en una de las cuales se encontraba Redfield.

En la mañana siguiente, partieron en tren a Lima, al barrio de Montserrate, para inspeccionar el enorme vagón de carga que les había provisto la Peruvian Corporation y que había sido transformado por el grupo norteamericano en un laboratorio rodante y con ayuda del personal y material provistos por la Grace Line. Después, se les concedió otro vagón, adicionado al tren, para que les sirviera de vivienda temporalmente. Esa noche, Barcroft revisó el programa de las tareas asignadas para cada investigador.

Al día siguiente, 20 de diciembre, a las 6:00 am, Redfield y Binger partieron hacia Cerro de Pasco. A las 5:30 pm, la locomotora, el vagón de depósito, el vagónlaboratorio y cuatro vagones de pasajeros partieron hacia Chosica, a 2800 pies (Figura 2). En su diario acotó: "Chosica es una avanzada de Lima...Aparte de las montañas que se levantan por encima de uno, hay mucho más que admirar en la naturaleza por debajo de ellas. Los dos recuerdos que llevo conmigo son los de una acacia violeta con una floración tan copiosa como codesos y las largas filas de caballos y burros. Algunos llevando leche, otros con pieles de vicuña. Los arneses de los caballos son muy hermosos".

El 21 de diciembre, a las 8:00 am, dejaron Chosica hacia Matucana (7 800 pies), continuaron a San Bartolomé y llegaron a la estación de Surco. Durante el viaje no se

\footnotetext{
${ }^{13}$ En 1920, Barcroft fue uno de los primeros en clasificar los estados anóxicos de la sangre por el contenido de oxihemoglobina. En 1932, JP Peters y DD van Slyke distinguieron el cuarto tipo de anoxia, citotóxica, producida por injuria a los mecanismos oxidativos celulares, como es el caso de la intoxicación por cianuro. En 1941, el término hipoxia sería introducido por C Wiggers.
} 


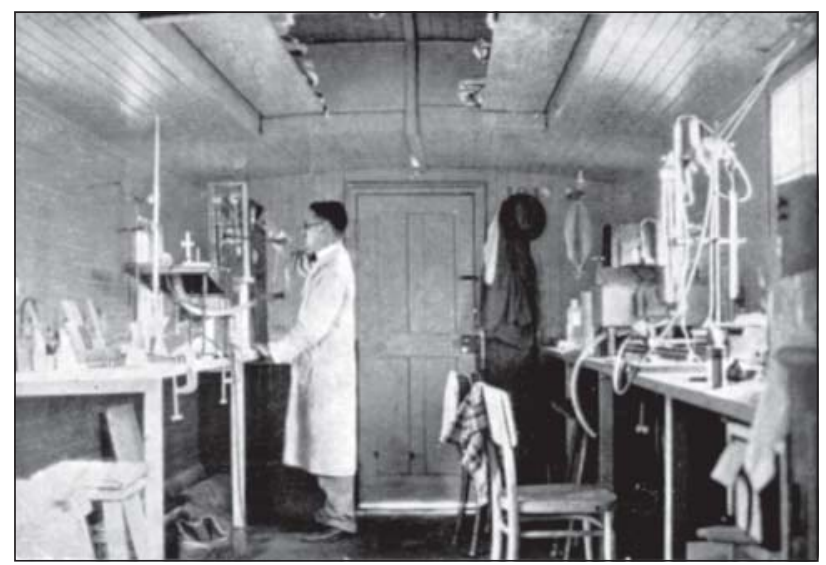

Figura 2. Interior del Vagón Laboratorio. Expedición de Barcroft 1921-2, Ferrocarril Central del Perú.

cansaron de admirar el paisaje, la flora silvestre y la fauna doméstica a ambos lados de la vía férrea: "Lo maravilloso de todo esto es que es imposible describirlo. Todo el tiempo hemos estado ascendiendo por el curso del río. El río tiene un torrente impetuoso que te dará una idea del gradiente del curso. Cuando el torrente forma una cascada, el gradiente se torna imposible de ascender y tenemos que hacer un zigzag. Así, hay uno en San Bartolomé; la estación es una cañada con inmensas montañas que la coronan e inmensos riscos por encima; tu vista se pasea por ellos y de repente puedes ver dos líneas férreas en la pared rocosa, la locomotora es desenganchada del tren y colocada en el otro extremo, y avanza en la dirección por la cual tú vienes, pero siempre yendo hacia arriba frente a la roca. Después de haber viajado por tal vez una hora, miras hacia abajo y ves el techo de la estación de San Bartolomé, abajo en el abismo. En Chosica, tuvimos algo inusual, llamado una noche húmeda, pero las circunstancias que siguieron le dieron una gran belleza a nuestro viaje, la atmósfera se tornó seca y las montañas más distantes estuvieron coronadas de nubes de color añil. El sol iluminó las más cercanas de la manera más bella. Ellas parecen ser completamente de granito, pero con un tinte amarillento parecido al granito de The Glen, en lugar del granito azulado de Bessbrook. Algunas veces, los bordes amarillentos se tornan rojos. Las sombras que variaron hasta un azul profundo, en la distancia, hicieron un paisaje maravilloso. En la ascensión, la vegetación aumenta. Aquí las laderas están cubiertas por tallos de pasto que sin duda serían muy verdes si no fueran tan empinadas para el agua subyacente...De San
Bartolomé hasta aquí hemos observado flores maravillosas. Un cactus crece como una columna de doce pies de altura, pienso, con una gran flor crema con forma de campana en la punta. Los pimientos son muy comunes, con sus frutos rojos, heliotropo, adelfa, yucas inmensas, higuera, plátanos y azucenas, son sólo algunas de las cosas que vimos desde la ventana. Antes de esto, en la estación de Surco, las mujeres estuvieron vendiendo los ramos de violetas más maravillosos. Pienso que ningún ramo tenía un diámetro menor de nueve pulgadas. Nunca he visto algo parecido." ${ }^{2}$ ).

El 23 de diciembre, el grupo partió a las 11.00 am hacia La Oroya, en el carro del Dr. H Crane, médico de la Cerro de Pasco Company. A las 5:00 pm, el tren pasó Ticllo (a 15000 pies) para descender hacia La Oroya (a 12200 pies). Allí conocieron al ingeniero jefe de la Cerro de Pasco Company, Mr. Colley. Barcroft enfermó de soroche, por lo que fue recostado en una cama del hospital, junto a Bock, quien se encontraba en peores condiciones (Figura 3).

El 24 de diciembre, Redfield, Meakins y Doggart partieron hacia Cerro de Pasco (14 200 pies). Barcroft y el resto del grupo recién partieron el 26 de diciembre, llegando a Cerro a las 9:30 pm, para alojarse en el hotel de la compañía y con prohibición de realizar ejercicios.

Para el 29 de diciembre, el grupo estuvo aclimatado y dejaron las instalaciones del hotel para establecerse en una casa con todas las comodidades. Pasaron la noche del Año Nuevo en una fiesta local. Los trabajos se realizaron a partir del 2 de enero. Pero, hay que resaltar

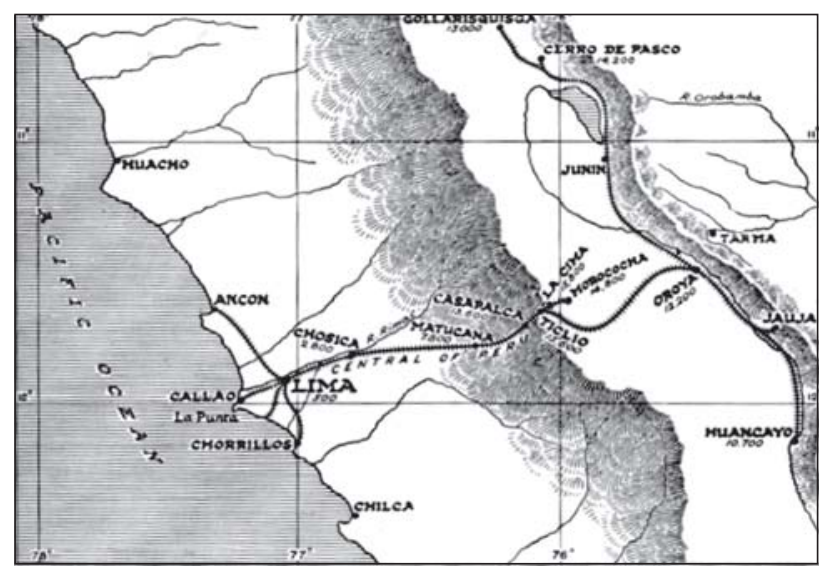

Figura 3. Mapa de la Región Central del Perú. Expedición de Barcroft a Cerro de Pasco, 1921-2. 
que las mediciones fueron realizadas desde diversas alturas antes de llegar a la zona y continuarían en el viaje de retorno a Norteamérica. El 5 de enero, Barcroft consideró que el grupo estaba trabajando demasiado y decidió darle un descanso. Redfield y Binger fueron a cazar patos al Lago Junín, mientras que el resto del grupo se dirigió a Gollarisquiga (13 000 pies), quedando impresionado por el paisaje ("Entonces, te das cuenta por primera vez que estás en el techo del mundo"). Retornaron a Cerro en la tarde del siguiente día. El 9 de enero, Barcroft consideró que lo programado se había cumplido. En los dos días siguientes se dedicaron a empacar, a culminar las tareas pendientes y las cosas menores.

El jueves 12 de enero, a las 7:00 am partieron en medio de una cordial despedida por las amistades hechas durante su permanencia. En La Oroya tuvieron otra despedida similar, partiendo a las 11.00 am y llegando a Casapalca (13 600 pies) a las 2:30 pm. Allí pernoctaron $\mathrm{y}$, al día siguiente, todo el grupo acompañado de $\mathrm{Mr}$. Campbell, gerente de la mina local y de Mr. Colley, realizaron un ascenso en mulas hacia la cima del monte Carlos Francisco (c. 17500 pies). Esa tarde, Bock y Harrop realizaron el retorno hacia Matucana en un carro manual para probar algunos respiradores antes de reunirse con el grupo al día siguiente.

El 13 de enero, todos hicieron el viaje de retorno hacia Lima. Aquí permanecieron hasta el 18 de enero de 1922, en que fueron trasladados al Callao para ser embarcados en el SS Ebro, de Belfast, de la SPNC, con destino a Nueva York, a través del canal de Panamá. En los días siguientes, durante la travesía, Barcroft asignó tareas para el procesamiento y tabulación de la información recogida.

Ya en Boston, el 27 de enero, dio la Conferencia Novell. El 2 de febrero llegó a Nueva York y el 11 de febrero dio la sexta conferencia Harvey de la Academia de Medicina sobre la razón de ser de los glóbulos rojos. Se embarcó el 1 de marzo para llegar el día 9 a Cambridge.

\section{¿Qué ocurría con la medicina nacional?}

Hacia fines de junio de 1919, el Grito de Córdoba había calado profundamente en el estudiantado universitario y se inició el movimiento de la Reforma Universitaria. La universidad peruana se encontraba en crisis: las autoridades de la Universidad Mayor de San Marcos habían sido declarados vacantes por resolución suprema en mayo de 1919. Los profesores civilistas se opusieron y la Universidad entró en receso. El 4 de julio de 1919, el presidente José Pardo y Barreda fue depuesto y asumió el gobierno don Augusto B. Leguía como Presidente Provisorio. Los universitarios entraron en huelga general el 31 de julio.

El 2 de octubre de 1919, la Asamblea Nacional eligió a Leguía como Presidente Constitucional. Los estudiantes obtuvieron notables reivindicaciones de parte del gobierno con los decretos 4002 y 4004 . En cumplimiento de estas normas, varias cátedras de la Facultad de Medicina bastión de los civilistas- fueron declaradas vacantes el 15 de noviembre. Esto motivó la renuncia de los profesores de medicina. Las concesiones del gobierno eran aparentes, pues buscaban enfrentar a los alumnos contra los profesores civilistas, lo que significó más confrontaciones entre los propios estudiantes, contra los profesores civilistas y contra la policía $\left.{ }^{5,6}\right)$.

El 30 de junio de 1920, Leguía promulgó la Ley Orgánica de Enseñanza. El 22 de marzo de 1921, un grupo de profesores recesó la Universidad de San Marcos.

En la primera legislatura de 1921, el 31 de mayo, el Congreso nombró una comisión para evitar que los estudiantes continuaran perdiendo con el receso y decretó la reorganización de la Universidad. El 12 de abril de 1922, el gobierno dio por terminada su intervención y la Universidad reabrió sus aulas. Para el período 1921-1922, el Dr. Ricardo L Flores era el decano de la Facultad de Medicina y el Dr. Ernesto Odriozola, el presidente de la Academia Nacional de Medicina.

En medio de este clima político, social y universitario hostil, con una facultad de medicina recesada, con dirigentes políticos y estudiantiles detenidos o deportados, llegó Barcroft al Perú liderando la expedición angloamericana.

Es interesante hacer notar que Barcroft no hizo ningún contacto con los médicos locales, la Facultad de Medicina de Lima o la Academia Nacional de Medicina. Eso sí, en la introducción de su trabajo, publicado posteriormente, agradeció al Gobierno Peruano, además de las instituciones privadas que le dieron facilidades. No existe alguna referencia sobre esta expedición en el diario El Comercio, los Anales de la Facultad de Medicina de Lima, la Crónica Médica, el Boletín de la Sociedad 
Geográfica de Lima de la fecha; e, incluso, no sería consignada en algunas relaciones importantes de viajeros extranjeros al Perú $\left({ }^{7,8}\right)$.

\section{RETORNO A CAMBRIDGE}

Durante el resto del año, Barcroft fue invitado, por lo menos una vez al mes, a dar una conferencia sobre su expedición al Perú. El 15 de diciembre de 1922, intervino en la discusión organizada por la Real Sociedad de Medicina sobre los aspectos médicos de la vida en las grandes alturas, donde participaron algunos integrantes de la expedición al Monte Everest, como TG Longstaff y TH Sommervell, y JS Haldane, dado la amplitud del tema.

El trabajo completo para publicación, sobre la expedición a los Andes, fue recibido el 25 de julio de 1922, pero no fue publicado sino hasta enero de 1923 (Figura 4). Dicho trabajo no tiene el formato actual para una publicación científica. Luego de la introducción, entra ordenadamente en el desarrollo de sus mediciones acompañadas de la discusión pertinente. Sus conclusiones fueron $\left({ }^{9}\right)$ :

1. la presión de oxígeno arterial fue siempre menor que la arterial, lo que sería explicable por difusión, aunque podría serlo accidentalmente por otro mecanismo.

2. Confirmó una vez más lo hallado por otros investigadores, que la ventilación total se incrementa en la altura, lo que produce disminución de la presión de $\mathrm{CO}_{2}$ y un incremento de la de oxígeno. Para tratar de explicar este resultado, midió las concentraciones del ión hidrógeno en la sangre en reposo y en actividad, sin llegar a conclusión alguna.

3. Que, ante una caída de la concentración de oxígeno alveolar, se incrementa la capacidad fijadora de oxígeno de la hemoglobina, es decir, la curva de disociación de la hemoglobina se desplaza a la derecha. Encontró datos contradictorios sobre si aumentaba la alcalemia. Ésta y el por qué del desplazamiento de la curva de disociación de la hemoglobina fueron dos grandes interrogantes que se hizo.

4. Realizando mediciones de la caja torácica en radiografías, concluyó que no hubo variaciones a nivel de mar y en Cerro entre los investigadores; sin embargo, éstos tenían una mayor proporción altura/ ancho de la caja torácica y una mayor pendiente de la $8^{\text {a }}$ costilla que los nativos.
5. Las determinaciones de la capacidad vital y del aire alveolar residual de los investigadores, a nivel del mar y una semana o más después en Cerro, no mostraron cambios importantes.

6. El coeficiente de difusión del monóxido de carbono tendió a incrementarse.

7. Hubo un incremento de la hemoglobina, la masa corpuscular roja y los reticulocitos durante el ascenso y entre los residentes.

8. No se encontró diferencias en la frecuencia del pulso a nivel de mar y en la altura; pero, con el ejercicio, la frecuencia se elevó en desproporción al esfuerzo realizado.

9. Existía un leve incremento del volumen minuto que estaría dentro de la variación esperada para el método empleado. Y el flujo sanguíneo en la mano fue el mismo que a nivel del mar.

10. Con el gasto sistólico se halló resultados contradictorios, dependiendo del método empleado; y no hubo variación en el tamaño de la silueta cardiaca a juzgar por las medidas tomadas en radiografías.

11. A pesar de las dificultades para medir el volumen sanguíneo, se encontró una discreta elevación, que fue correlacionada con la temperatura ambiental.

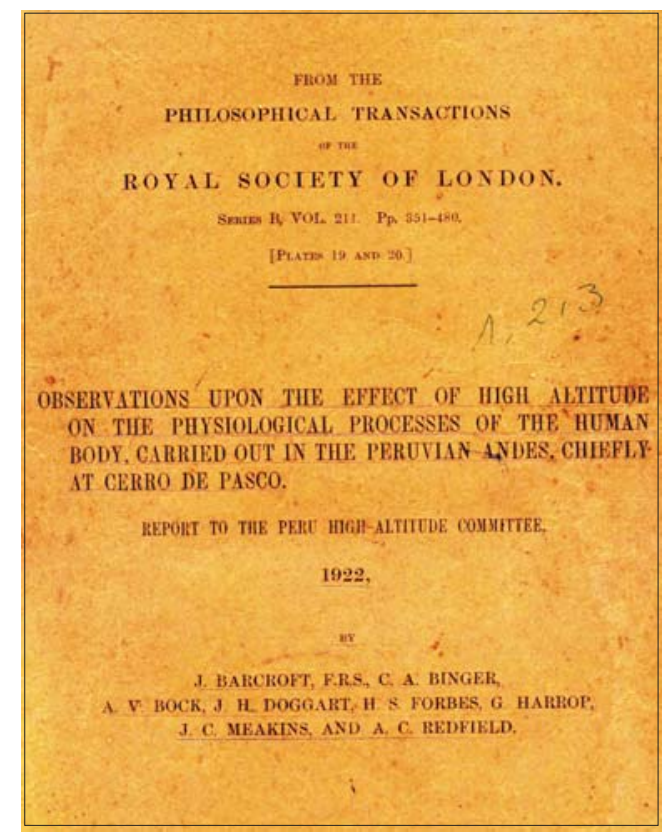

Figura 4. Carátula de la publicación de Barcroft et al en Philosophical Transactions,1922. 
12.El coeficiente de difusión (diferencia de la presión promedio de oxígeno en el aire alveolar menos la presión promedio arterial de oxígeno/ consumo de oxígeno por minuto) se encontró disminuido.

13. La cianosis es un hallazgo frecuente en la altura, especialmente luego del ejercicio.

14. Las pruebas mentales ordinarias que se hizo entre los investigadores no mostraron incapacidad alguna; sin embargo, reconocieron cierta falta de concentración en sus trabajos. Concluyeron que hubo cierta fatiga mental y física y que tal vez las pruebas no eran las apropiadas.

15. La conclusión final fue: “...la principal contribución que hemos hecho es la observación de que, en nuestro equipo de trabajo, la propensión al soroche corresponde muy cercanamente al valor del coeficiente de difusión; aquellas personas que tenían un coeficiente de difusión bajo fueron las que más sufrieron."

En las sucesivas conferencias que Barcroft dio en 1922, sostuvo que, efectivamente, había una enfermedad por la menor concentración de oxígeno en los tejidos, que mejoraba inmediatamente con el descenso. Que la adaptación de los nativos se debía a: 1) un incremento total de la ventilación pulmonar que elevaba la presión parcial de oxígeno y disminuía la presión parcial del dióxido de carbono; 2) una marcada expansión de la caja torácica, muy evidente en los nativos, leve en los norteamericanos y ausente en los visitantes; 3) un incremento de la masa globular roja y de la hemoglobina; $y, 4)$ una afinidad incrementada de la sangre por el oxígeno. Que la importancia relativa de estos cuatro factores estaba por ser determinada y que la susceptibilidad a contraer el mal de montaña dependía de la variación individual en la constante de difusión, como había sido sugerido por Krogh. Que concentrarse fue muy difícil en Cerro de Pasco y que los empleados mineros podían sostener escasa actividad mental por períodos largos a 14000 pies. Que ningún miembro de la expedición pudo dormir bien en las grandes alturas. Que al final del mes, todos ellos se sintieron mentalmente exhaustos.

En realidad, la experiencia de Barcroft y col sobre la expedición a los Andes tiene muchas limitaciones en su diseño como trabajo de investigación. Actualmente, dicho trabajo no habría sido aprobado en una revista científica. No tenía un objetivo bien definido. La casuística era escasa: las mediciones fueron hechas entre uno y seis expedicionarios, en tres personas de la compañía minera $\mathrm{y}$ en tres o cuatro nativos. No fue uniforme: las mediciones fueron incompletas muchas veces. Sin embargo, a pesar de los pocos casos y resultados incompletos, hizo grandes generalizaciones.

Barcroft siguió trabajando y publicando sobre otros aspectos de fisiología y dando conferencias sobre los problemas de altura. Precisamente, en la discusión mencionada del 15 de diciembre, Haldane dijo que Barcroft había remecido la evidencia aportada por su expedición al Monte Pikes, que la actividad secretoria de oxígeno incrementada por parte del epitelio pulmonar era el factor principal de la aclimatación; pero, en todo caso, el porcentaje de saturación de la hemoglobina era desproporcionado a la disminución de la presión parcial del oxígeno alveolar.

En octubre de 1923, Barcroft recibió la Medalla Baly, que se entregaba bienalmente al personaje más distinguido en las ciencias fisiológicas. A fines de ese año, publicó sobre la captación de CO por la hemoglobina en el bazo y sobre el efecto de la temperatura externa sobre la circulación en humanos. Al año siguiente, se dedicó a sus trabajos sobre las características de la hemoglobina.

En 1925, publicó The respiratory function of the blood Part I. Lessons from high altitude, en el que resumió su experiencia en los Andes. El 16 de diciembre de 1925, se le adjudicó el cargo de Profesor de Fisiología dejado por Langley, quien había fallecido el 12 de noviembre pasado. ${ }^{14}$

Barcroft continuó investigando sobre el volumen sanguíneo circulante, el lecho capilar sanguíneo y las propiedades de la hemoglobina; y, entre sus numerosas conferencias, destacaron sus trabajos acerca de la función del bazo. Fue, precisamente, a través de los trabajos experimentales sobre las alteraciones del volumen de bazo en la gestación, que se fue interesando por la fisiología circulatoria y respiratoria de la gestación y de los fetos.

\footnotetext{
${ }^{14}$ John Newport Langley (1852-1925). Segundo profesor de Fisiología de Cambridge, fue muy conocido por su contribución al desarrollo de la Escuela Médica de Cambridge, por haber sido el editor del Journal of Physiology y por sus numerosos trabajos sobre la estructura y función del sistema nervioso autónomo.
} 
El Dr. Carlos Monge Medrano había iniciado sus trabajos sobre el habitante de las alturas en $1927 . .^{15}$ Es muy probable que Monge se hubiera enterado de la expedición angloamericana en los primeros meses de 1922, cuando ella se encontraba en los Andes peruanos. Es también probable que Monge haya tenido conocimiento de las conclusiones de Barcroft, entre 1923 y 1924. Según el propio Monge, él ya enseñaba el problema de la desadaptación en las grandes alturas, basado en su experiencia, en el curso de Nosografía Médica, en 1924, y que había presentado en un informe ante la Academia Nacional de Medicina, en 1925. Hasta ese momento, Monge estuvo interesado en otros temas, como puede observarse al revisar su producción bibliográfica.

Cuando llegó Barcroft, Monge era profesor interino de Nosografía Médica de la Facultad de Medicina y jefe del Laboratorio de Sanidad del Hospital Militar de San Bartolomé. En 1925, fue jefe del Servicio de Medicina del Hospital Arzobispo Loayza; y, en 1927, fue jefe del Servicio de Medicina Interna del Hospital Militar de San Bartolomé. En mayo de 1929, Monge asistió al Congreso de Medicina Interna en Londres, como delegado del gobierno peruano, pero no consta que se haya entrevistado con Barcroft $\left({ }^{10}\right)$.

Ante una supuesta conclusión de Barcroft de que "el nativo de la altura no podrá ser físicamente ni mentalmente igual al del nivel del mar", Monge reaccionó preparando una expedición a la sierra central, en 1927, para demostrarle lo contrario. ${ }^{16}$ Para Monge, "Es sensible que los miembros de la expedición angloamericana, Barcroft y sus asociados, no conocieran este síndrome del que ellos padecieron sin saberlo, como se deduce de la lectura de sus protocolos y las observaciones consignadas en las páginas 445, 446 y 453, donde se asegura que el hombre aclimatado no existe, y que los habitantes de la altura son personas de poder mental y físico restringido (impaired), generalizando o todos los que ellos habían experimentado." $\left.{ }^{11}\right)$
En realidad, Barcroft nunca hizo semejante aseveración inicialmente, lo cual se corrobora con la lectura del trabajo original $\left({ }^{9}\right)$. Es en Lessons fron high altitude donde se encuentra el comentario de que "El hombre aclimatado no es aquel que ha logrado un poder físico y mental, tanto en Cerro de Pasco como lo sería en Cambridge (sea Massachussets o Inglaterra). Tal hombre no existe. Todos los habitantes de las grandes alturas son personas con poderes físicos y mentales disminuidos." ${ }^{13}{ }^{17}$ Esta referencia es citada por Monge varias veces en lo sucesivo y es la que realmente dio sustento a su empeño en demostrarle lo contrario a Barcroft.

Este fue el aserto que motivó a Monge y a los investigadores nacionales y que ha continuado generando opiniones hasta la actualidad: "Esta afirmación debe haber sido penosa para el espíritu peruano y científico de Monge. Dos terceras partes de los peruanos vivían en la altura desde hacían miles de años; habían creado y mantenido sabias y florecientes culturas; habían trabajado las minas y el campo; habían desarrollado sus alegres y extenuantes danzas; y habían sostenido infatigables la rudeza de los deportes. No podía caber en el pensamiento de Monge, buscador privilegiado de la verdad, tan extraña conclusión aparentemente científica. El hombre de los Andes conmovía con agudeza su espíritu desde antes." (sic) $\left({ }^{14}\right)$.

Enhorabuena, pues la reacción de Monge significó muchos trabajos de investigación sobre los diversos procesos fisiológicos y patológicos del habitante de las alturas andinas en los siguientes años. Inclusive, podemos afirmar que la corriente científica médica impuesta por Monge se hallaba dentro de la gran corriente indigenista, muy en boga por cierto. ${ }^{18}$ Este indigenismo científico médico podemos verlo como una respuesta al darwinismo social y los movimientos eugenistas que imperaban en la época. De lo que sí podemos estar seguros, a deducir de la vida y obra de Barcroft, es que su posición fue eminentemente científica y sin ningún atisbo de racismo.

\footnotetext{
${ }^{15}$ Carlos Monge Medrano (1884-1970). Iniciador de la biología y patología andina. Describió la desadaptación a la altura (enfermedad de Monge). Decano de la Facultad de Medicina, presidente de la Academia Nacional de Medicina, rector interino de la Universidad Mayor de San Marcos.

${ }^{16}$ Este proceso de reacción por los médicos nacionales ante el accionar de los investigadores extranjeros en nuestro territorio ha sido una característica de la medicina peruana $\left({ }^{12}\right)$.

${ }^{17}$ Barcroft, Joseph. The respiratory function of the blood Part I. Lessons from high altitude. Cambridge, University Press, 1925 : 176. (Op. cit. por Monge).

${ }^{18} \mathrm{Si}$ bien es cierto que el proceso denominado ‘indigenismo’ está referido primariamente a resaltar la condición humana del indio en la literatura y pintura nacional, desde fines del siglo XIX y primeras década del siglo XX, Monge estudia al indio de tal manera que intentará demostrar que se trata de la "raza con el mayor rendimiento físico del mundo".
} 
El 22 de marzo de 1927, en la reunión de la Sociedad Literaria y Filosófica de Manchester, Barcroft dio la Conferencia Wilde sobre "Fisiología de la vida en las alturas de los Andes», y allí sostuvo que la enfermedad de la montaña era un descubrimiento del Nuevo Mundo; y, con respecto a su experiencia en los Andes peruanos, dijo "Nunca un europeo está completamente bien allí, logra sobreponerse a la falta de aire y a las palpitaciones de su corazón en gran medida, pero nunca alcanza un vigor real sea físico o mental." ${ }^{19}$

Monge se refirió con respecto a Barcroft como que «sin darse cuenta, estuvo afectado con la enfermedad subaguda del soroche». En efecto, Barcroft padeció el soroche a su arribo a La Oroya y tres días después ya se había aclimatado, tal como lo anotó en su diario de viaje. Barcroft también observó que los lugareños podían jugar a la pelota sin problema alguno y que los ingenieros norteamericanos se desempeñaban normalmente, pero que tenían que tomar largos momentos de descanso. Su aseveración fue en el sentido de que un europeo estaría en desventaja física y mental en las grandes alturas, pero también creía que su aseveración era válida para el habitante andino ${ }^{20}$. Los diversos estudios posteriores le dieron la razón a Barcroft. Esto es cierto hoy, aunque existan algunas creencias como aquella de que hay que tolerar las molestias de la altura porque es una cuestión de 'machos' o cuando se pretende negar que los ambientes enriquecidos de oxígeno en los centros de trabajo resultaran más ventajosos $\left({ }^{4,15}\right) .{ }^{21}$

Por otro lado, 'aclimatación' significaba, y significa, para los fisiólogos anglosajones aquellos cambios que se producen en el organismo al llegar a la altura. Para Monge, estos cambios corresponden a adaptación; y, para él, aclimatación significa los cambios presentes en el organismo del individuo que nace en la altura (congénita) o que vive por muchos años en ella (adquirida) ${ }^{16}$ ).

Barcroft se enteró, en 1927, sobre el trabajo de Monge, pero no le contestó. Para él, y continuó sosteniéndolo cada vez que exponía sobre el tema, el hombre europeo y el habitante de las alturas sometido a una baja presión de oxígeno ambiental, no tendría el mismo vigor físico y mental que a nivel del mar. En realidad, lo que había sido uno de los objetivos de su viaje ya estaba zanjado: se había impuesto la teoría de la difusión pasiva o mecánica a través de la membrana alveolar. ${ }^{22} \mathrm{Y}$, para cuando Monge estudiaba con ahínco los cambios fisiológicos en los habitantes de la altura, Barcroft investigaba sobre la transferencia de los gases en los lechos capilares, especialmente en el uterino. Esto devendría, en los años siguientes, en sus trabajos sobre la fisiología placento-fetal.

$\mathrm{Al}$ igual que Barcroft, los fisiólogos ingleses de esa época sólo estuvieron interesados en los aspectos básicos de la fisiología respiratoria y sus variaciones en las condiciones existentes en las grandes alturas. Con excepción de esta parte del continente, y principalmente gracias a los investigadores peruanos, poco se había estudiado sobre la enfermedad derivada de la exposición a las grandes alturas. El sesgo de los investigadores de los países desarrollados a no buscar referencias y estudios de los países latinoamericanos, ha contribuido a que, salvo excepciones, la repercusión o impacto de estas publicaciones haya sido mínimo. Después de la Segunda Guerra Mundial, apareció un inusitado interés por el montañismo (alpinismo y andinismo) que despertó la necesidad de conocer esta enfermedad.

Recién en los últimos 30 años es que se viene estudiando científicamente el diagnóstico, tratamiento y

\footnotetext{
${ }^{19}$ Pág, 169. Op. Franklin.

${ }^{20}$ En realidad, lo que decía Barcroft no era novedad. Denis Jourdanet, médico francés que había residido varios años en México, en 1861, publicó Les altitudes de l'Amerique tropicale comparées au niveau des mers au point de vue de la constitution médicale y, en 1863, en el artículo l'anemie des altitudes et de l'anemie en général dans ses rapports avec la pression de l'atmosphere, publicado en la Revue Médicale, expuso su teoría de la anoxihemia barométrica, según la cual los habitantes de las grandes alturas tienen un menor rendimiento físico y mental debido a la 'anemia cerebral' que se produce al respirar un aire con menor concentración de oxígeno.

${ }^{21}$ Los efectos fisiológicos de la hipoxia de la altura son numerosos. En definitiva, el consumo máximo de oxígeno en los tejidos está disminuido, el rendimiento mental también está disminuido y el sueño se encuentra alterado. Esto es sostenido por investigadores de la talla de John West.

${ }^{22}$ La cosa no es tan simple. Con los actuales conocimientos de la ultraestructura de la célula, sus membranas compuestas por capas de proteínas y lípidos favorecen, como se sostiene ahora, un complejo mecanismo a nivel molecular para el traslado de sustancias, un transporte biológicamente activo en lugar de la simple difusión mecánica. Esta es la 'teoría del mosaico fluido', desarrollada alrededor de $1970\left({ }^{1}\right)$.
} 
prevención del mal de montaña agudo, es decir, la enfermedad derivada de la exposición a la altura (edema cerebral, edema pulmonar) $\left({ }^{17}\right)$. Sobre la desadaptación a la altura o mal de montaña crónico, que fue la gran temática de Monge, se escribió muy poco en otras latitudes, tanto así que se le sigue considerando como propio del habitante andino, a diferencia del tibetano $\left({ }^{4}\right)$.

En 1931, Barcroft publicó dos trabajos experimentales sobre el efecto de la temperatura en la frecuencia cardiaca y respiratoria con José Joaquín Izquierdo, fisiólogo e historiador de la medicina mexicano, quien hizo una pasantía en Cambridge.

En 1932, partió hacia Sudamérica para dar unas conferencias en Río de Janeiro (Brasil) y en Buenos Aires (Argentina). En esta última ciudad, fue recibido por el Profesor de Fisiología Bernardo A. Houssay ${ }^{23}$ y su asistente, el Dr. Alfredo Biasotti. Tuvo un cálido recibimiento, una recargada agenda de actividades académicas y sociales, entre el 19 y 31 de agosto, quedando gratamente encantado. Fue hecho miembro honorario de la Sociedad de Biología de Argentina y de la Academia Nacional de Medicina. Se dirigió a Montevideo para partir luego hacia Lisboa.

Ya en Cambridge, publicó sus trabajos acerca del efecto de las lesiones accidentales sobre el tamaño del bazo, las alteraciones en el tamaño del bazo denervado durante la gestación, sobre el volumen sanguíneo del útero durante la gestación y sobre los lechos sanguíneos (bazo, útero, hígado, piel). Uno muy particular fue el referido a algunas observaciones sobre la porción proximal del colon exteriorizado.

A inicios de 1933, Barcroft dejó de ser Profesor de Fisiología de la Universidad de Cambridge y publicó su trabajo sobre el incremento del flujo sanguíneo y del metabolismo gaseoso en el útero gestante.
A mediados de 1933, viajó a los Estados Unidos invitado como uno de los 75 científicos más distinguidos, con motivo del World's Fair obtenido por la ciudad de Chicago. En Nueva York, visitó el Instituto Rockefeller y fue recibido por Donald D van Slyke ${ }^{24}$, entre otras personalidades. Se dirigió a Toronto, donde departió con Fitzgerald, Banting, Best, Taylor y Frazer, entre otros. Ante la Asociación Norteamericana de Ciencias dio su conferencia sobre las condiciones de la respiración fetal. De regreso a Europa, recibió el grado honorario de Doctor en Ciencias de la Universidad Nacional de Dublín.

En 1934, publicó su libro Features in the Architecture of Physiological Function, donde dio a conocer los principios de sus experimentos. Este libro fue escrito de una manera muy especial, integrando los diversos órganos, aparatos y sistemas y no como una simple sucesión de ellos $\left({ }^{18}\right)$. Hacia fines de ese año, en coautoría con otros investigadores, empezó a publicar sus trabajos experimentales en conejas, ovejas y cabras, sobre la respiración fetal.

A partir de 1935, y hasta la segunda gran guerra mundial, tuvo como un cercano colaborador al Dr. Donald H Barron. El 10 de julio de 1935, fue investido por el Rey George V como Caballero de la Corona Inglesa, en el Palacio de Buckingham. En agosto de ese año, concurrió al Congreso Internacional de Fisiología que se llevó a cabo en Leningrado y Moscú. Aquí tuvo la oportunidad de conocer al eminente fisiólogo ruso, Pavlov ${ }^{25}$.

En marzo de 1936, falleció el gran fisiólogo John Scott Haldane ${ }^{26}$, lo que significó un hondo pesar para Barcroft. En agosto de ese año partió nuevamente hacia los Estados Unidos, invitado para la celebración del tricentenario de Harvard. Luego, se dirigió a Toronto, donde fue recibido por Charles H Best.

En Cambridge, continuó trabajando con Barron, especialmente sobre diversos aspectos de la fisiología y

\footnotetext{
${ }^{23}$ Beranrdo Alberto Houssay (1887-1971). Fisiólogo argentino. En 1947, ganó el Premio Nóbel de Medicina y Fisiología, que compartió con Carl Cori y Gerty Cori, el que le fue adjudicado por su contribución al estudio de la interrelación de la glándula hipófisis con el metabolismo de la glucosa.

${ }^{24}$ Donald Dexter van Slyke (1883-1971). Bioquímico norteamericano que realizó importantes estudios en patología renal, diabetes mellitas y los trastornos ácido-básicos. Creó aparatos y métodos para determinar la eliminación de urea y la concentración del bicarbonato en la sangre.

${ }^{25}$ Ivan Petrovich Pavlov (1849-1936). Premio Nóbel de Medicina y Fisiología, en 1904, por sus estudios sobre la fisiología de la digestión.

${ }^{26}$ John Scott Haldane (1860-1936). Fisiólogo británico. Demostró que el $\mathrm{CO}_{2}$ controla el mecanismo de la respiración (1905); estudió el efecto de la altura y la presión del agua sobre la respiración y fabricó una cámara para descompresión de los buzos; mejoró las condiciones de trabajo de los mineros expuestos a altas concentraciones de monóxido de carbono y del uso de los equipos de rescate; fundó el Journal of Hygiene y estudió el golpe de calor.
} 
los movimientos somáticos del feto. En este año fue nominado para el premio Nóbel, pero éste fue conferido a Henry Dale y Otto Loewi.

En 1937, ya con 65 años, dejó la Cátedra de Fisiología, cargo que fue ocupado por Adrian ${ }^{27}$. En los tres años siguientes, continuó trabajando bajo el auspicio de fondos asignados por el Consejo de Investigación Médica para investigadores reconocidos (1935-1936 y 1936-1937) y también para asistir a investigadores de Cambridge (1937-1938 y 1938-1939).

En 1939, en los días de haberse iniciado la Segunda Guerra Mundial, junto con Barclay, Barron y Franklin publicaron los estudios radiográficos de la circulación a través del corazón adulto, del corazón fetal y del ductus arteriosus; y empezó los estudios funcionales del cierre de cortocircuitos circulatorios fetales, como el ductus arteriosus y el foramen ovale.

Su producción se redujo notablemente durante los años de la guerra. Formó parte del Comité de Guerra Química. En 1940, publicó sus trabajos experimentales en carneros acerca del aporte de oxígeno al cerebro fetal y el efecto de la asfixia (oclusión del cordón umbilical) sobre los movimientos respiratorios, y del contenido de oxígeno en los vasos umbilicales; en 1941, sobre la capacidad de oxígeno de la sangre fetal y neonatal y sobre los cambios de la circulación fetal después del nacimiento. En este año, fue invitado a formar parte de la naciente Sociedad de Nutrición y de de la Escuela de Fisiología Veterinaria, en conexión con el Consejo de Investigación Agrícola, ambas con necesidades para responder a urgencias con la producción y provisión de alimentos durante la guerra.

Los numerosos trabajos que Barcroft publicó con Barron sirvieron para que ambos fueran considerados posteriormente como los "Padres de la fisiología fetoplacentaria". ${ }^{28}$

Barcroft publicaba, por un lado, sobre el inicio de la respiración al nacer y, por otro, mostraba sus resultados sobre el proceso de desecado de la carne y la leche. Y participaba en conferencias sobre la alimentación de la población en tiempos de guerra y sobre los elementos trazas en relación con la salud. ${ }^{29}$

Al año siguiente, después de sus trabajos sobre la función nutritiva de la placenta, es de resaltar la demostración de la disminución del contenido de oxígeno en los senos venosos, por punción intracraneal, en fetos de conejos posmaduros. Por estos trabajos de fisiología fetal, Barcroft recibió la Medalla Copley de la Real Sociedad (1943) y fue nombrado Miembro Honorario por el Real Colegio de Obstetricia y Ginecología (1944). La Real Sociedad de Edimburgo también le concedió similar distinción (1944). Barcroft fue distinguido como profesor honorario de seis universidades europeas y norteamericanas.

Por esta época, junto con sus colaboradores, se interesó en la producción de ácidos volátiles por la microflora intestinal, especialmente en el intestino grueso de los rumiantes. Hacia fines de 1946, salió publicado su último libro Researches on Pre Natal Life (Investigaciones sobre la Vida Prenatal), Volumen I, dedicado al Dr. Barron por sus notables contribuciones, sin restar méritos de sus otros colaboradores como JD Boyd, Alfred Ernest Barclay y Kenneth J Franklin.

Con estas líneas de trabajo, Barcroft continuó trabajando y disertando hasta que, el 21 de marzo de 1947, falleció repentinamente de un infarto cardíaco. Cuatro días más tarde sus restos fueron cremados. Su muerte significó una gran pérdida para sus discípulos, colaboradores, amistades y familiares. Su memoria continuó reafirmándose en los diferentes eventos sobre fisiología, nacionales e internacionales, de los cuales Barcroft siempre fue un asiduo concurrente con sus trabajos originales.

Barcroft fue un científico muy meticuloso con el diseño y preparación de sus experimentos. Es muy probable que haya sido un fiel seguidor del llamado 'Principio de Krogh': "Para cualquier problema siempre

\footnotetext{
${ }^{27}$ Edgar Douglas Adrian (1889-1977). Por sus notables trabajos en el campo de la electrofisiología del sistema nervioso central y periférico había recibido el Premio Nóbel en 1932.

${ }^{28}$ Donald Henry Barron (1905-1993). Biólogo norteamericano. Después de una destacada carrera como biólogo, anatomista, neurofisiólogo y fisiólogo feto-placentario en las universidades de Cambridge, Missouri y Yale, fue nombrado Profesor Emérito en esta última. En 1969, ocupó la cátedra de Medicina y Biología Reproductiva en la Universidad de La Florida, iniciando una segunda carrera. Es considerado también como el "Padre de la obstetricia científica".

${ }^{29}$ En el Octavo Congreso de la Sociedad de Nutrición, que se realizó en la Escuela de Higiene y Medicina Tropical de Londres, Barcroft sugirió que debería definirse a los elementos trazas (trace elements): son elementos metálicos -con excepción del flúor y del yodo-, activos en el organismo; existen en las cantidades adecuadas -su exceso o su déficit se traducen en daño-, presentes en trazas -no más de 0,00005 partes- y su acción es como la de una enzima.
} 
hay un animal en el cual pueda estudiarse de la manera más conveniente." $\left({ }^{19}\right)$. Su pensamiento estuvo inmerso en el más puro mecanicismo reduccionista de la escuela germana que imperaba allá por los años $1920\left({ }^{20}\right)^{30}$.

Barcroft fue una persona muy amable, afable, con gran sentido del humor, gran conversador, presto a hacer amistades y un notable conferencista. Inspiró afecto a sus discípulos y colegas, quienes lo recordarían con gratitud. Un gran creyente de la santidad de la familia y del hogar. También gustó de la lectura, pintura, equitación, navegación y el golf. Modesto y generoso en el crédito de las autorías con sus co-investigadores $\left({ }^{21}\right)$.

Existe una montaña que lleva su nombre: el Monte Barcroft, dentro de la montaña White-Inyo, California, de 13040 pies (3 975 m). Allí se estableció, en 1950, el Laboratorio de Investigaciones de Altura de la Universidad de California, el cuarto más alto de su género en Norteamérica (12 470 pies), que es visitado frecuentemente por ecólogos, astrofísicos, biólogos y geólogos.

\section{AGRADECIMIENTOS}

Al Dr. Róger Guerra García Cueva, por permitirme acceder al libro de Kenneth J Franklin y a un ejemplar original (separata) del trabajo de Barcroft de la biblioteca de la Cátedra Alberto Hurtado de la Universidad Peruana Cayetano Heredia.

\section{REFERENCIAS BIBLIOGRÁFICAS}

1. Agutter PS, Malone PC, Wheatley DN. Diffusion theory in biology: a relic of mechanistic materialism. J Hist Biol. 2000;33(1):71-111.

2. Franklin KJ. Joseph Barcroft 1872-1947. Oxford: Blackwell Scientific Publications; 1953.

3. Samaja M. Blood gas transport at high altitude. Respiration. 1997;64(6):422-8.

4. West JB, American College of Physicians, American Physiological Society. The physiologic basis of high-altitude disease. Ann Intern Med. 2004;141(10):789-800.

5. Gamarra JM. El Desarrollo del movimiento reformista. En: La Reforma Universitaria. Lima: Okura Editores SA; 1987. p. $150-88$

6. Pacheco A. El gobierno de la universidad en el Perú. Lima: Fondo de Desarrollo Editorial de la Universidad de Lima; 1997. p. 81-120.

7. Díaz S. Índice Analítico del Boletín de la Sociedad Geográfica de Lima, Tomo I al CIV, Años 1891 a 1984. Serie Opúsculos sobre Geografía del Perú, Lima: Ed. S.E. Antúnez de Mayolo R.; 1988.
8. Núñez E. Viajes y Viajeros Extranjeros por el Perú. Lima: CONCYTEC; 1989.

9. Barcroft J, Binger CA, Bock AV, Doggart JH, Forbes HS, Harrop G, Meakins JC, Redfield AC. Observations upon the effect of high altitude on the physiological proccesses of human body, carried out in the Peruvian Andes, chiefly at Cerro de Pasco. Philos Trans Roy Soc Lond Biol Sci. 1923; 211:351-454.

10. Lerner D. Carlos Monge, Cronología. En: Carlos Monge, Obras. Compilación de Dora Lerner de Bigio. Tomo I. Lima: Universidad Peruana Cayetano Heredia y CONCYTEC; 1988. p. XXI-XXX.

11. Monge C. La Enfermedad de los Andes. An Fac Med Lima. 1928;XI(1-2):1-13.

12. Pamo O. La contribución extranjera en el desarrollo de la medicina peruana. En: Temas de la Historia Médica del Perú. CONCYTEC. Lima: Megaprint Ediciones SA.; 1990. p. 95129.

13. Monge C. Acclimatization in the Andes. Introduction. Baltimore: The Johns Hopkins Press; 1948. p. XI-XIX.

14. Velásquez MT. Prólogo. En: Carlos Monge, Obras. Compilación de Dora Lerner de Bigio. Tomo I. Lima: Universidad Peruana Cayetano Heredia y CONCYTEC; 1988. p. VIII.

15. West JB. Acclimatization to high altitude: truths and misconceptions. High Alt Med Biol. 2003;4(4):401-2.

16. Monge MC. La Enfermedad de los Andes. Segunda parte. Cap V. Adaptación-Aclimatización-Desadaptación. An Fac Med Lima. 1928;XI(1-2):76-106.

17. Milledge JS. High Altitude Medicine: A British Perspective. High Alt Med Biol. 2003;4(3):261-4.

18. Roberts KB. The Principal structures of Physiology: what and where are they? The case of Barcroft's architecture. Am J Physiol. 1998;274(6 Pt 2):S6-17.

19. Lindsten J. Schack August Steenberg Krogh - A versatile genius [monografía en el Internet]. Stockholm: The Nobel Foundation; 2005 [citado el 20 junio de 2005]. Disponible en: http://nobelprize.org/medicine/articles/ $\mathrm{krogh} / \mathrm{s} / \mathrm{f}$.

20. Bischof M. Some remarks on the history of Biophysics (and its future). En: Zhang CL, Popp FA, Bischof M (editors). Current Development of Biophysics. Hangzhou: Hangzhou University Press; 1996.

21. Dunn PM. Sir Joseph Barcroft of Cambridge (1872-1947) and prenatal research. Arch Dis Child Fetal Neonatal Ed. 2000;82(1):F75-6.

Manuscrito recibido el 10 de marzo de 2005 y aceptado para publicación el 02 mayo de 2005.

Correspondencia: Dr. Oscar G. Pamo Reyna

Facultad de Medicina, UNMSM. Av. Grau 750. Lima 1, Perú.

Correo-e: oscarpamo@terra.com.pe

\footnotetext{
${ }_{30}$ Jacques Loeb (1859-1924), fisiólogo norteamericano nacido en Prusia que emigró a los EE.UU. en 1891. Autor de The mechanistic conception of life (1912). Su filosofía reduccionista propugnaba que todos los procesos vitales, incluyendo la voluntad y la conducta humanas, pueden ser explicados por simples procesos mecánicos.
} 